\title{
Composite Materials Based on Foam Polyurethane and Graphene Nanoplates Effectively Screening Electromagnetic Radiation
}

\author{
N.R. Memetov ${ }^{1}$, A.V. Gerasimova ${ }^{*}$, R.A. Stolyarov ${ }^{1}$, A.G. Tkachev ${ }^{1}$, \\ A.V. Melezhik ${ }^{1}$, N.A. Chapaksov ${ }^{1}$, A.S. Osipkov ${ }^{2}$, P.A. Mikhalev ${ }^{2}$, A.S. Provatorov ${ }^{2}$ \\ ${ }^{1}$ Department of Technology and Methods of Nanoproducts Manufacturing, \\ Tambov State Technical University, 1, Leningradskaya ul., Tambov, 392000, Russia; \\ ${ }^{2}$ Bauman Moscow State Technical University (National Research University of Technology), \\ bld. 1, 5,. 2nd Baumanskaya ul., Moscow, 105005, Russia \\ *Corresponding author. Tel.: +7 95312206 42.E-mail: alyona_gerasimova_92@mail.ru
}

\begin{abstract}
Composite materials based on polyurethane foam and graphene nanoplates (GNP) for shielding from electromagnetic radiation in the frequency range from 2 to $12 \mathrm{GHz}$ were made by impregnation. The use of phenol-formaldehyde resin (PFS) as a binder component promoted good adhesion of graphene to the polymer matrix and made it possible to obtain samples of composites with a high graphene concentration of $\sim 50 \mathrm{wt} \%$. An increase in the shielding efficiency of composites was found both with an increase in the frequency of electromagnetic radiation and with an increase in the concentration of GNP in them. The maximum shielding value was $75 \mathrm{~dB}$ at a frequency of $12 \mathrm{GHz}$ and graphene concentration of $50 \%$ by mass. It is concluded that the impregnation method proved to be promising for the manufacture of flexible and lightweight composite foams effective for shielding electromagnetic interference in the range from 2 to $12 \mathrm{GHz}$.
\end{abstract}

\section{Keywords}

Nanocomposite; graphene nanoplates; electromagnetic radiation; phenol-formaldehyde resin; shielding coefficient; polyurethane foam.

(C) N.R. Memetov, A.V. Gerasimova, R.A. Stolyarov, A.G. Tkachev, A.V. Melezhik, N.A. Chapaksov, A.S. Osipkov', P.A. Mikhalev, A.S. Provatorov, 2020

\section{Introduction}

Due to the widespread use of various electrical and electronic devices in everyday life and work, pollution by electromagnetic interference is becoming a serious problem today, since they have an adverse effect on human health [1-4]. Of particular importance is the problem of individual protection of people when they perform various jobs in conditions of high-frequency electromagnetic radiation (EMR). Depending on the frequency, the EMR has a different penetration depth into various materials. The human body is no exception [1]. As a result, certain tissues and organs can be exposed to an electromagnetic field (EMF) [1, 3, 4]. When EMR is absorbed, the temperature of the object rises. For biological objects, and especially for humans, an increase in temperature by $1-5$ degrees can cause numerous malformations, temporary infertility in men, brain damage and changes in the chemical composition of blood [1]. Even a slight increase in temperature by about $1{ }^{\circ} \mathrm{C}$ in the human body can lead to a change in hormone production and suppression of the immune response $[1,5]$.

Of course, the most effective solution to the problem is to combat the cause, namely, reducing the intensity of electromagnetic radiation. Thus, the European Union funded the project "Networks with a low level of exposure to electromagnetic fields" (LEXNET) [6] under the FP7 program, in order to reduce at least $50 \%$ the impact on the population of electromagnetic fields without compromising the quality of communication and data transfer [2].

However, there are areas where it is impossible to exclude the effect of electromagnetic radiation on humans and biological objects. Therefore, the urgent task is to develop materials that can be used in personal protective equipment against electromagnetic radiation. Materials should effectively shield EMI (SE EMI of at 
least $20 \mathrm{~dB}$ ), be lightweight and flexible [7]. Using foamed polymer composites, it is possible to create shielding products with high service characteristics compared to materials based on metal [7-9]. To ensure high shielding characteristics, it is necessary that the polymer material possesses high electrical conductivity $[7,10]$. Among non-metallic fillers, carbon nanotubes (CNTs) and graphene are the most effective, since possess the highest values of electrical conductivity in comparison with other carbon nanostructures $[11,12]$. The main advantage of graphene over nanotubes is its high reflectivity of EMR, which is preserved when used in polymer composites as a shielding component [13].

The use of absorbing EMR materials, in particular nanotubes [14-17], in personal protective equipment will cause them to heat up, and therefore, discomfort during human use. CNTs are also rather difficult to evenly distribute in a polymer matrix because of their confusion $[18,19]$. Therefore, graphene nanoplates can be effectively used as a modifying component, since they are efficiently distributed in most polymer matrices, can be a concomitant surfactant (in oxidized form) [20], and can also provide shielding properties for polyurethane matrices. To achieve a high degree of shielding, it is necessary to increase the concentration of graphene in polyurethane foam, as was shown in [7-9]. Therefore, the development of production methods and the study of highly concentrated polyurethane foam composites is relevant.

Thus, the aim of the work was to obtain a highly effective shielding material based on polyurethane foam and graphene nanoplates.

\section{Method}

To study the shielding from electromagnetic radiation, five polyurethane foam samples were prepared with a mass graphene content of $10,20,28$, 40 , and $50 \%$ by weight of the polyurethane foam.

The procedure for obtaining these samples consisted of the following stages.

\section{Obtaining GNP modified with phenol-formaldehyde resin (GNP / FFS)}

An experimental batch of water paste GNP / FFS was used, manufactured by Ltd NanoTechCenter (Russia, Tambov). Manufacturing technology includes oxidative intercalation of natural graphite GSM-2, mixing intercalated graphite with an aqueous solution of phenol-formaldehyde resin type Fenotam GR-326 (non-volatile residue $50 \%$, production - Krata PJSC, Tambov, Russia), ultrasonic treatment. Under these conditions, at least partial grafting of oligomeric FFS molecules to the surface of oxidized graphene were occurred (under these processing conditions, graphene nanoplates contain $10-13 \%$ of oxide groups). The degree of exfoliation of graphite to graphene during ultrasonic processing was controlled by the light absorption coefficient of samples diluted with water according to the procedure described in [21]. After ultrasonic treatment, the suspension was filtered to a paste state.

In the resulting aqueous paste, the mass content of graphene was 5.5-6.0\%, the content of PFS 30-50\% by weight of graphene. Typical images of the obtained GNP in a scanning and transmission electron microscope are shown in Fig. 1.

\section{Obtaining samples of polyurethane foam, modified GNP / FFS}

Polyurethane foam plates of $10 \times 220 \times 220 \mathrm{~mm}$ in size were used as the substrate material. The initial mass of such a plate was about $8.3 \mathrm{~g}$.

The GNP / FFS paste was diluted with a small amount of water and processed on a homogenizer for several minutes, which allowed to obtain a homogeneous suspension. The mass of graphene paste was chosen so that the amount of dry graphene in it was $10,20,28,40$, and $50 \%$ of the mass of the initial sample of polyurethane foam. The amount of water for diluting graphene paste was chosen so that the entire solution was absorbed into the pores of the polyurethane foam without residue. After impregnation, the samples were dried in a stream of hot air under a fan heater, rotating the sample all the time so that the impregnating solution would not flow in one direction. The completeness of the drying was controlled by weight (up to constant weight). Due to the presence of the phenol-formaldehyde component acting as glue, the samples prepared in this way hold graphene firmly, it does not get dirty and does not crumble upon mechanical action on the sample.

After impregnation and drying, the thickness of the samples was about $7 \mathrm{~mm}$, i.e., the samples shrinked compared to the initial thickness of $10 \mathrm{~mm}$. 


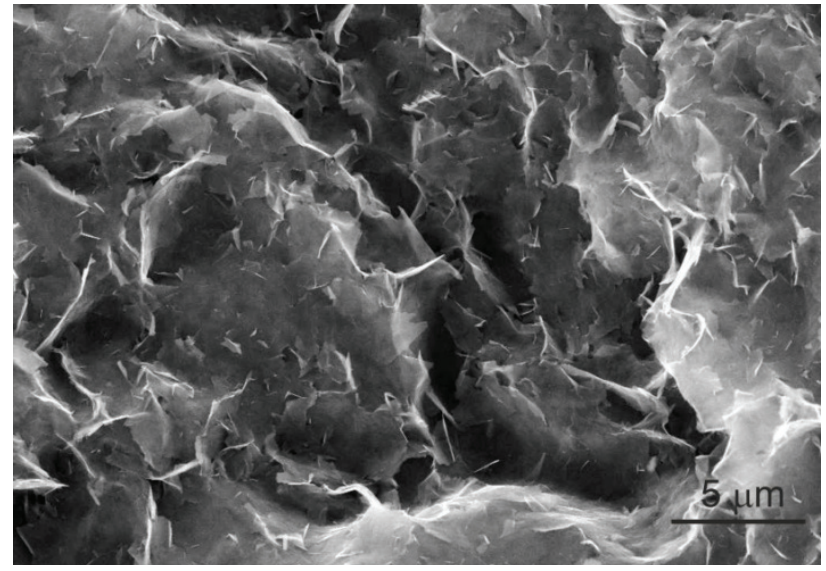

a)

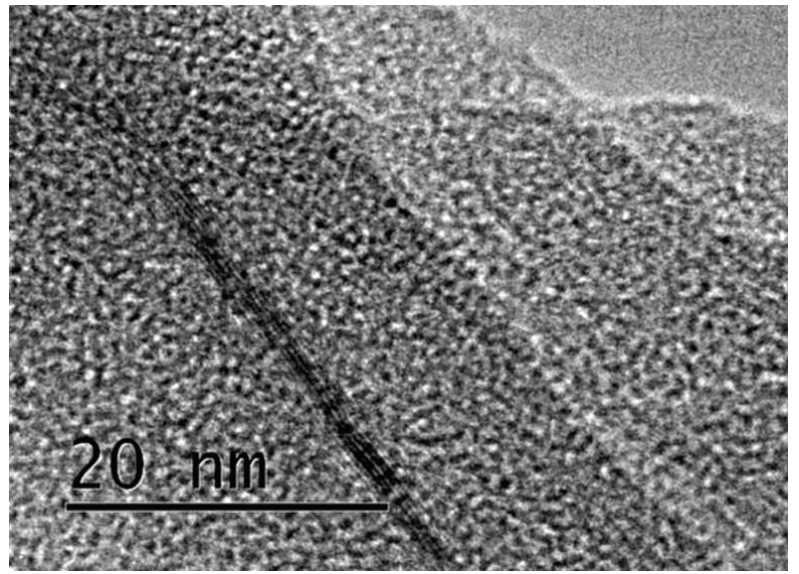

b)

Fig. 1. Images of graphene nanoplates modified with FFS: $a-\mathrm{SEM} ; b-\mathrm{TEM}$

\section{Characterization and research methods}

The morphology and microstructure of the GNP surface, as well as individual plates, were studied using a Carl Zeiss Merlin scanning electron microscope (SEM) and a JEOL JEM 2100F transmission electron microscope (TEM).

The electrodynamic characteristics (screening and reflection coefficients) of composite polyurethane foams were measured on a measuring stand (Fig. 2) in accordance with the procedure described in [22]. In this case, filtering of noise in the time domain was used.

When measuring the screening coefficient (CE), the transmitting antenna was A1, the receiving one was A2. To determine the FE, reference measurements were carried out: without installing the test sample (empty window) and with the installation of a metal diaphragm of sufficient thickness, and measurement with the installation of the test sample in the equipment of the measuring stand. CE (SE) was determined by the ratio:

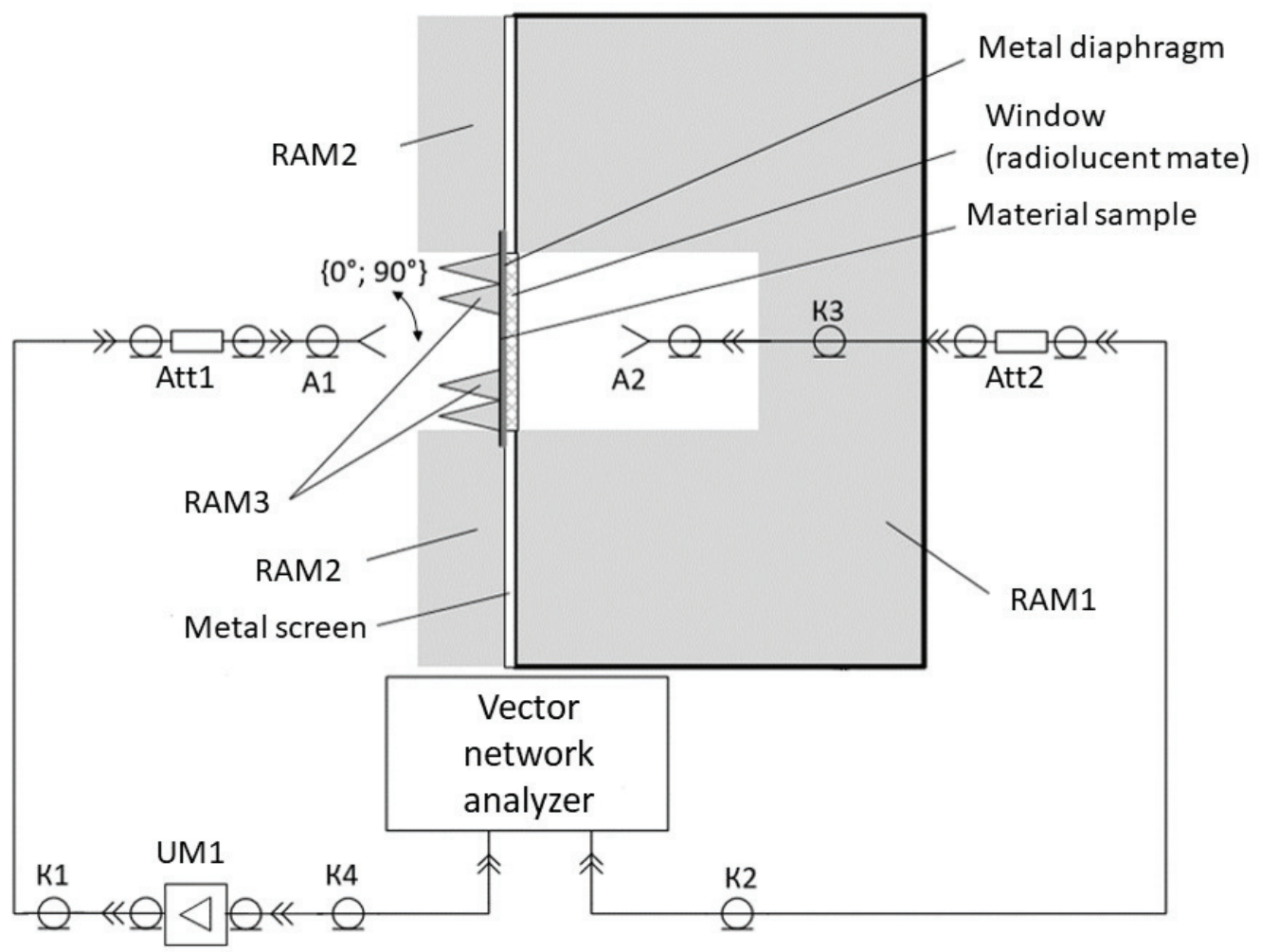

Fig. 2. Diagram of a measuring stand for studying the electromagnetic characteristics of samples 


$$
\mathrm{SE}=-20 \lg \left|\left(S_{21, \mathrm{obr}}-S_{21, \mathrm{~m}}\right)\right|+20 \lg \left|\left(S_{21, \mathrm{v}}-S_{21, \mathrm{~m}}\right)\right|,
$$

where $S_{21 \text {,obr. }}$ - complex transfer coefficient with the test sample installed in the equipment; $S_{21, \mathrm{~m}}$ - complex transfer coefficient with a metal screen of sufficient thickness installed in the equipment; $S_{21, \mathrm{v}}$ - complex transfer coefficient with an empty window.

When measuring the reflection coefficient on metal $R_{\text {Me }}$, a sample of material was laid on a sheet of metal of sufficient thickness. The measurements were carried out on a single antenna $\mathrm{A} 1$, which worked on the transmission and reception. To determine $R_{\mathrm{Me}}$, reference measurements were carried out: without installing the test sample (empty window) and with the installation of a sheet of metal of sufficient thickness, and measurement with the installation of the test sample on a sheet of metal of sufficient thickness. $R_{\mathrm{Me}}$ was determined by the ratio:

$$
R_{\mathrm{Me}}=20 \log \left|\frac{U_{\mathrm{obr}}-U_{\text {fon }}}{U_{\mathrm{Me}}-U_{\text {fon }}}\right| \text {, }
$$

where $U_{\text {obr }}$ - the complex value of parameter $S_{11}$ for a sample on a metal sheet; $U_{\mathrm{Me}}$ - the complex value of parameter $S_{11}$ for a sheet of metal; $U_{\text {fon }}$ - the complex value of parameter $S_{11}$ for an empty measuring cell.

The value $R_{\mathrm{Me}}$ characterizes the loss of electromagnetic energy in the sample.

\section{Results and discussion}

Fig. 3 shows the dependences of the screening coefficient SE of the samples on the EMR frequency.

An increase in the efficiency of shielding of composites was found both with an increase in the frequency of electromagnetic radiation and with an increase in the concentration of GNP in them.

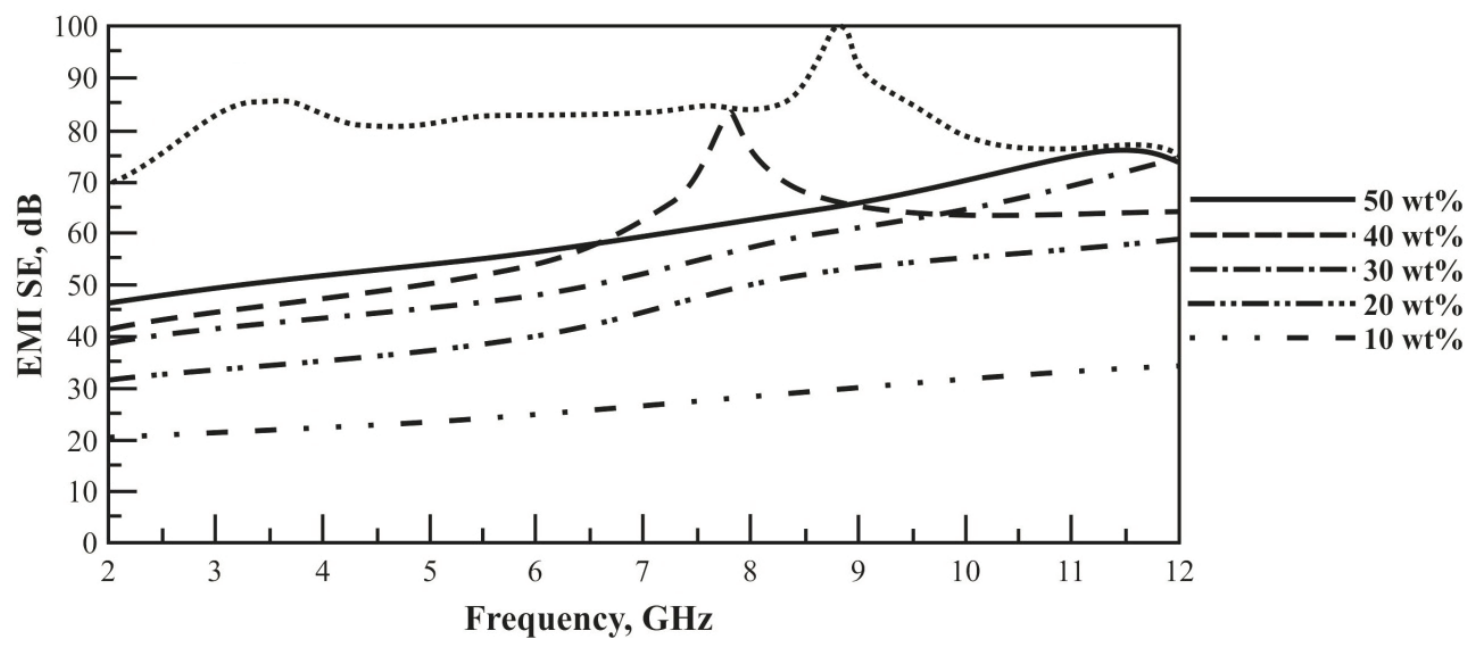

Fig. 3. Screening coefficient dependences (EMI SE) of the studied samples of polyurethane foam composites on the frequency of EMR

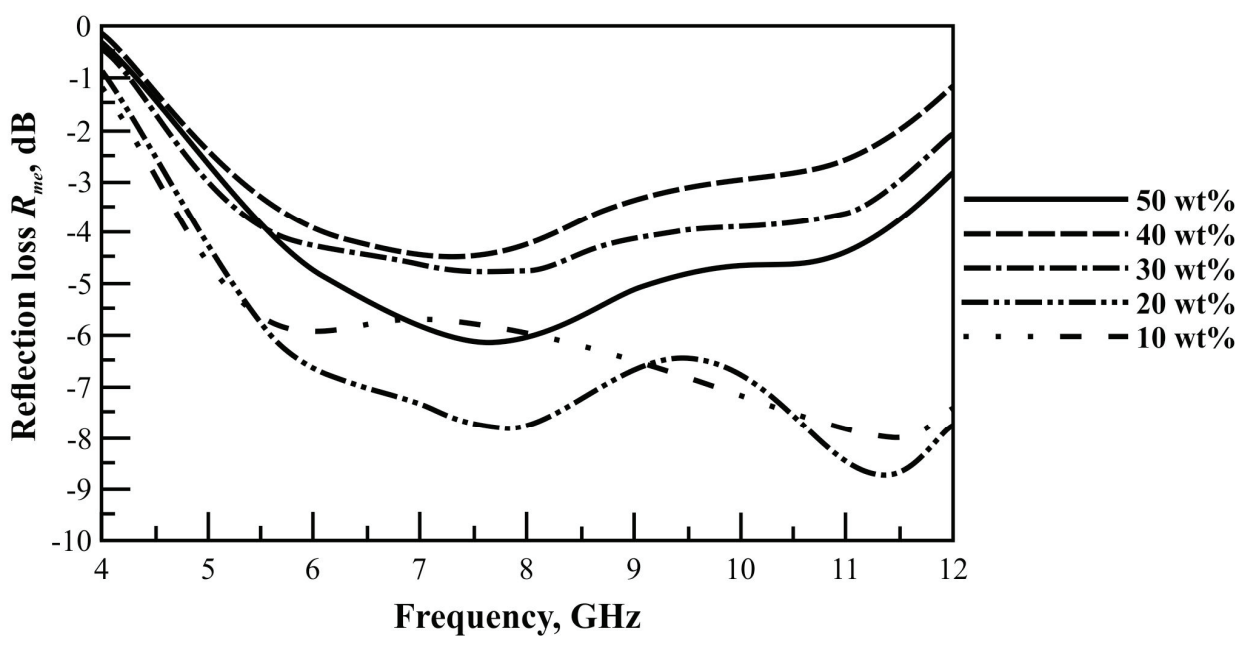

Fig. 4. Reflection coefficient dependences on the metal $\boldsymbol{R}_{\mathrm{Me}}$ of the studied samples of polyurethane foam composites on the frequency of electromagnetic radiation 
Table 1 low absorbing properties and high shielding effect,

Comparative characteristics of shielding foamed polymer composites (X EMR frequency range)

\begin{tabular}{lcccc}
\hline Polymer & $\begin{array}{c}\text { Graphene } \\
\text { content, wt } \%\end{array}$ & $\begin{array}{c}\text { Thickness, } \\
\mathrm{mm}\end{array}$ & $\begin{array}{c}\text { EMI SE, } \\
\mathrm{dB}\end{array}$ & References \\
\hline TPU & 50 & 7.0 & 75 & This work \\
TPU & 6.5 & 1.8 & 21.8 & {$[7]$} \\
TPU & 20 & 2.4 & 20 & {$[9]$} \\
PS & 30 & 2.5 & 29 & {$[23]$} \\
PMMA & 5 & 2.4 & 19 & {$[24]$} \\
PEI & 10 & 2.3 & 13 & {$[25]$} \\
\hline
\end{tabular}

An increase in graphene concentration logically leads to an increase in the screening coefficient (Fig. 3), which is probably associated with an increase in the electrical conductivity of the samples [7, 10]. The maximum shielding value was $75 \mathrm{~dB}$ at a frequency of $12 \mathrm{GHz}$ at a graphene concentration of $50 \%$ by mass.

To study the absorption capacity of material samples, studies of the coefficient $\mathrm{R}_{\mathrm{Me}}$ of reflection on the metal were conducted, the results of which are presented in Fig. 4.

As can be seen from the dependency graphs (Fig. 4), the resulting composites have low absorption characteristics ( $R_{\mathrm{Me}}$ values do not exceed minus $9 \mathrm{~dB}$ ). In a compartment with high SE values, this indicates that the studied samples of materials mainly reflect EMR, which opens up prospects for their use in personal protective equipment.

Table 1 compares the shielding characteristics for the samples obtained in our work and existing analogues (foamed polymer composites).

Obtained material has comparable shielding characteristics compared to analogues (Table 1), and in some cases surpasses them, however, the developed method for producing foamed polyurethane composites is simpler.

\section{Conclusion}

Composite materials based on polyurethane foam and graphene nanoplates were successfully fabricated using a simple impregnation method. With an increase in the GNP content in polyurethane foam, the screening coefficient of the samples increased. The screening coefficient increases from 45 to $75 \mathrm{~dB}$ with an increase in the frequency of electromagnetic radiation from 2 to $12 \mathrm{GHz}$.

As a result, a technique was developed for producing light gas-permeable composites that have which can be successfully used in personal protective equipment against electromagnetic radiation and other applications.

\section{Acknowledgements}

We express gratitude to the senior scientist I.A. Damaratsky affiliated with N.E. Bauman MSTU for the assistance in taking measurements of the electrodynamic characteristics of materials.

The present study was funded by the Russian Foundation for Basic Research (RFBR) in the framework of Project No. 18-29-19121.

\section{References}

1. Wessapan T., Rattanadecho Ph. Temperature induced in human organs due to near-field and far-field electromagnetic exposure effects. International Journal of Heat and Mass Transfer, 2018, 119, 65-76.

2. Pasquino N. Measurement and analysis of human exposure to electromagnetic fields in the GSM band. Measurement, 2017, 109, 373-383.

3. Ennamany R., Fitoussi R., Vie K., Rambert J., Benetti L., Mossalayi M.D. Exposure to Electromagnetic Radiation Induces Characteristic Stress Response in Human Epidermis. Journal of Investigative Dermatology, 2008, $128,743-746$.

4. Jooyan N., Goliaei B., Bigdeli B., Faraji-Dana R., Zamani A., Entezami M., Mortazav S.M.J. Direct and indirect effects of exposure to $900 \mathrm{MHz}$ GSM radiofrequency electromagnetic fields on $\mathrm{CHO}$ cell line: Evidence of bystander effect by non-ionizing radiation. Environmental Research, 2019, 174, 176-187.

5. Stuchly M.A. [Health effects of exposure to electromagnetic fields]. Proceedings of the IEEE Aerospace Applications Conference, 1995, 1, 351-368.

6. Low EMF Exposure Future Networks (LEXNET). http://www.lexnet-project.eu.

7. Jiang Q., Liao X., Li J., Chen J., Wang G., Yi J., Yang Q., Li G. Flexible thermoplastic polyurethane/reduced graphene oxide composite foams for electromagnetic interference shielding with high absorption characteristic. Composites Part A, 2019, 123, 310-319.

8. Wang Ch., Li J., Guo Sh. High-performance electromagnetic wave absorption by designing the multilayer graphene/thermoplastic polyurethane porous composites with gradient foam ratio structure. Composites Part A, 2019, 125, 1-13.

9. Li Y., Shen B., Yi D., Zhang L., Zhai W., Wei X., Zheng W. The influence of gradient and sandwich configurations on the electromagnetic interference shielding performance of multilayered thermoplastic polyurethane/graphene composite foams. Composites Science and Technology, 2017, 138, 209-216.

10. Komarov F.F., Milchanin, O.V., Parfimovich, I.D., Grinchenko, M.V., Parhomenko, I.N., Tkachev, A.G., 
Bychanok, D.S. Absorption and Reflectance Spectra of Microwave Radiation by an Epoxy Resin Composite with Multi-Walled Carbon Nanotubes. Journal of Applied Spectroscopy, 2017, 84, 596-602.

11. Lekawa-Raus A., Patmore J., Kurzepa L., Bulmer J., Koziol K. Electrical properties of carbon nanotube based fibers and their future use in electrical wiring. Advanced Functional Materials, 2014, 24, 3661-3682.

12. Wang J., Ma F., Liang W., Sun M. Electrical properties and applications of graphene, hexagonal boron nitride (h-BN), and graphene/h-BN heterostructures. Materials Today Physics, 2017, 2, 6-34.

13. Parfimovich I.D., Komarov F.F., Milchanin O.V., Tkachev A.G., Schegolkov A.V. Microwave characteristics of a composite material based on epoxy polymer with the addition of graphene and ferrite nanomaterials. Reports $N A S B, 2019,63(1), 22-28$.

14. Komarov F.F., Tkachev A.G., Milchanin O.V., Parfimovich I.D., Grinchenko M.V., Parhomenko I.N., Bychenok D.S. A composite based on epoxy polymer and carbon nanotubes: structure, optical properties and interaction with microwave radiation. Advanced Materials and Technologies, 2017, 2, 19-25.

15. Kolanowska A., Janas D., Herman A.P., Jedrysiak R.G., Gizewski T., Boncel S. From blackness to invisibility - Carbon nanotubes role in the attenuation of and shielding from radio waves for stealth technology. Carbon, 2018, 126(31). doi.org/10.1016/j.carbon.2017.09.078

16. Basuli U., Chattopadhyay S., Nah C., Chaki T.K. Electrical properties and electromagnetic interference shielding effectiveness of multiwalled carbon nanotubes reinforced EMA nanocomposites. Pol. Comp, 2012, 33, 897. https://doi.org/10.1002/pc.22167

17. Zeng Z., Jin H., Chen M., Li W., Zhou L., Zhang Z. Lightweight and Anisotropic Porous MWCNT/WPU Composites for Ultrahigh Performance Electromagnetic Interference Shielding. Advanced Functional Materials,2015, 26, 303. https://doi.org/10.1002/adfm.201503579;
18. Yuen S.M., Ma C.C.M., Chuang C.Y., Yu K.C., Wu S.Y., Yang C.C., Effect of processing method on the shielding effectiveness of electromagnetic interference of MWCNT/PMMA composites. Compos Sci Technol, 2008, 68, 963-968.

19. Kim K., Cho S.J., Kim S.T., Chin I.-J., Choi H.J. Formation of two-dimensional array of multiwalled carbon nanotubes in polystyrene/poly(methyl methacrylate) thin film. Macromolecules, 2005, 38, 10623-10626.

20. Okhay O., Gonçalves G., Dias C., Ventura J., Vieira E.M.F., Goncalves L.M.V., Tkach A. Tuning electrical and thermoelectric properties of freestanding graphene oxide papers by carbon nanotubes and heat treatment. Journal of Alloys and Compounds, 2019, 781, 196-200.

21. Melezhyk A.V., Kotov V.A., Tkachev A.G. Optical Properties and Aggregation of Graphene Nanoplatelets. Journal of Nanoscience and Nanotechnology, 2016, 1, 10671075.

22. Marvin C., Dawson L., Flintoft I.D., Dawson J.F. A Method for the Measurement of Shielding Effectiveness of Planar Samples Requiring No Sample Edge Preparation or Contact Andrew. IEEE Transactions on Electromagnetic Compatibility, 2009, 51(2), 255-262.

23. Yan D.-X., Ren P.-G., Pang H., Fu Q., Yang M.-B., Li Z.-M. Efficient electromagnetic interference shielding of lightweight graphene/polystyrene composite. J Mater. Chem., 2012, 22(36), 18772-18774.

24. Zhang H.-B., Yan Q., Zheng W.-G., He Z., Yu Z.-Z. Tough grapheme - polymer microcellular foams for electromagnetic interference shielding. ACS Appl Mater Interfaces, 2011, 3(3), 918-24.

25. Ling J., Zhai W., Feng W., Shen B., Zhang J., Zheng Wg. Facile preparation of lightweight microcellular polyetherimide/graphene composite foams for electromagnetic interference shielding. ACS Appl Mater Interfaces, 2013, 5(7), 2677-84. 